\title{
Sistema remoto de alimentacion semiautomatizado para mascotas
}

\section{Semi-automatic remote feeding system for pets}

\author{
ZAPIEN-RODRIGUEZ, Jose Manuel $\dagger^{*}$, CRUZ-BRICEÑO, Guadalupe Janeth, ROSILES-REYES, \\ Carlos Andres y TORRES-CARRILLO, Eva Alejandra
}

Universidad Politécnica de Lázaro Cárdenas, Ingeniería Mecatrónica - Ingeniería Mecatronica. Av. Galeanas Sin Número, Colonia Las 600 Casas, C.P. 60950. Cd. Lázaro Cárdenas, Michoacán

ID $1^{\text {er }}$ Autor: Jose Manuel, Zapien-Rodriguez / ORC ID: 0000-0001-7198-2118, CVU CONACYT ID: 228342

ID $1^{\text {er }}$ Coautor: Guadalupe Janeth, Cruz-Briceño

ID $2^{\text {do }}$ Coautor: Carlos Andres, Rosiles-Reyes

ID $3^{\text {er }}$ Coautor: Eva Alejandra, Torres-Carrillo

DOI: $10.35429 /$ JCA.2020.14.4.26.31

Recibido Marzo 30, 2020; Aceptado Junio 29, 2020

\section{Resumen}

La sobrecarga de actividades de las personas propicia la falta de tiempo para el cuidado de las mascotas, lo que provoca que no reciban su alimento en horarios adecuados, o el caso contrario de solo dejar una torre de alimento sin medir proporciones. Esto ocasiona problemas graves en la salud de las mascotas, ya sea desnutrición u obesidad. El objetivo del proyecto es alimentar a las mascotas de manera adecuada en horarios establecidos, evitando problemas de salud debido a una mala dieta en la mascota por falta de tiempo, así mismo se evitará el desperdicio de alimento al darle porciones específicas de acuerdo a la toma de decisión automática por peso y tamaño de raza. Este proyecto se realizará con materiales reciclados, reduciendo el costo de elaboración. Una base que sostendrá un aspa de aluminio, esta será la encargada de girar permitiendo u obstruyendo el paso del alimento. En la parte superior se tiene un recipiente con tapa para almacenar el alimento. Con ayuda de una aplicación creada para celulares donde se ingresa el peso de tu mascota y el tamaño de su raza, se calcula la cantidad de alimento que le corresponde, este cálculo está basado en una tabla de alimento. En esta aplicación también se podrá establecer horarios diferentes para las tres comidas diarias.

Automatizacion, Aplicaciones moviles, IOT

\begin{abstract}
The overload of activities of the people causes the lack of time for the care of the pets, which causes that they do not receive their food in adequate schedules, or the opposite case of only leaving a food tower without measuring proportions. This causes serious problems in the health of pets in the long term, either malnutrition or obesity. The objective of the project is to feed the pets adequately at set times, avoiding health problems due to a bad diet in the pet due to lack of time, likewise avoiding the waste of food by giving specific portions according to the intake Automatic decision by weight and race size. This project will be carried out with recycled materials, reducing the cost of processing. A base that will hold an aluminum blade, this will be responsible for turning allowing or obstructing the passage of food. In the upper part there is a container with lid to store the food. With the help of an application created for cell phones where you enter the weight of your pet and the size of its breed, the amount of food that corresponds to it is calculated, this calculation is based on a food table. In this application you can also set different schedules for the three daily meals.
\end{abstract}

Citación: ZAPIEN-RODRIGUEZ, Jose Manuel, CRUZ-BRICEÑO, Guadalupe Janeth, ROSILES-REYES, Carlos Andres y TORRES-CARRILLO, Eva Alejandra. Sistema remoto de alimentacion semiautomatizado para mascotas. Revista de Cómputo Aplicado. 2020. 4-14: 26-31.

\footnotetext{
* Correspondencia al Autor (Correo Electrónico: zapien_jomazaro@uplc.edu.mx)

$\dagger$ Investigador contribuyendo como primer autor.
} 


\section{Introducción}

Este proyecto está dirigido a perros y gatos principalmente, sin embargo, se puede adaptar a otro tipo de mascota. Cuyo objetivo es alimentar a las mascotas de manera adecuada en horarios establecidos, evitando problemas de salud debido a una mala dieta en la mascota por falta de tiempo, así mismo se evitará el desperdicio de alimento al darle porciones específicas de acuerdo a la toma de decisión automática por peso y tamaño de raza.

En la actualidad disponemos me una menor cantidad de tiempo para alimentar a las mascotas de manera adecuada en horarios establecidos, evitando problemas de salud debido a una mala dieta en la mascota por falta de tiempo, así mismo se evitará el desperdicio de alimento al darle porciones específicas de acuerdo a la toma de decisión automática por peso y tamaño de raza.

La sobrecarga de actividades de las personas propicia la falta tiempo para el cuidado de las mascotas, lo que provoca que no reciban su alimento en horarios adecuados, o el caso contrario de solo dejar una torre de alimento sin medir proporciones. Esto ocasiona problemas graves en la salud de las mascotas a largo plazo, ya sea desnutrición u obesidad.

Anteriormente se han realizado proyectos similares presentando en diferentes documentos como tesis, artículos en congresos, los cuales se pueden tomar ideas en un futuro para mejorar el proyecto y lo más importante es la relevancia con la que cuentan proyectos de esta índole, tales como Diseño y construcción de un dosificador de Granos secos (Oviedo Navas, 2012), Comedero Automatizado para Pollos de Engorde (Sarmiento \& Vargas, 2014), Diseño Concurrente y Fabricación de un Dosificador Automático de Alimento para Mascotas (Consuegra M. \& González D., 2011), Desarrollo de un sistema de Dosificación Automático de Alimento para Equinos (FRANCO, GALICIA, \& OSTRIA, 2010). Dichos trabajos abordan el tema del diseño de los circuitos electrónicos y especifican los datos técnicos de algunos componentes.

\section{Equipo y materiales empleados}

El servo MG996R Tower Pro es robusto, de alto rendimiento, compacto, con engranes de metal, tamaño estándar, incluye accesorios y tornillos para montaje en cualquier proyecto. Este servo destaca por su buen torque $(11 \mathrm{Kg})$, por lo que es utilizado principalmente en proyectos de robótica, como brazos robóticos y robots bípedos. Puede rotar aproximadamente 180 grados $\left(90^{\circ}\right.$ en cada dirección). Tiene la facilidad de poder trabajar con diversidad de plataformas de desarrollo como Arduino, PICs, Raspberry Pi, o cualquier microcontrolador.

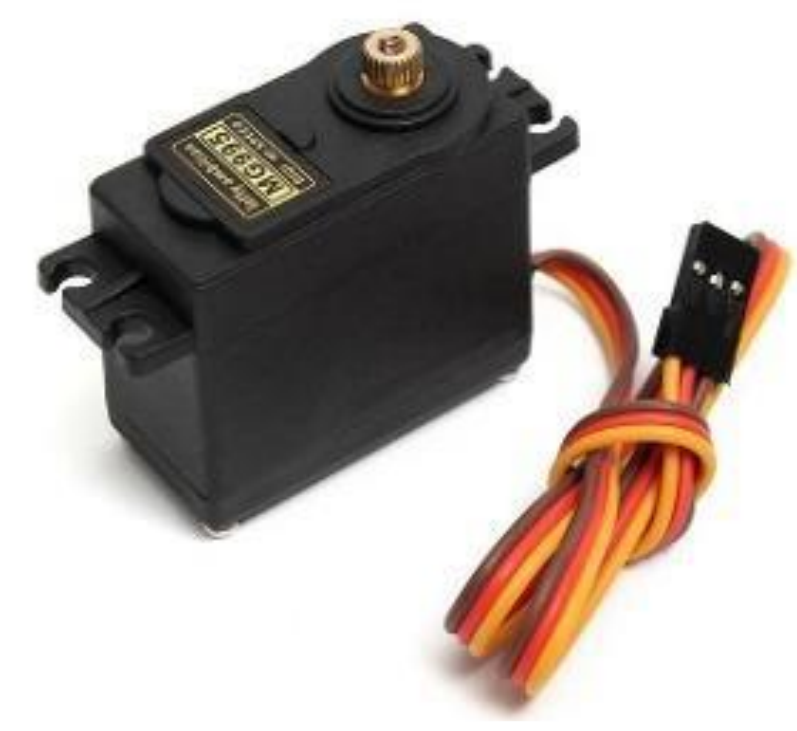

Figura 1 Servo MG996R Tower Pro

La celda de carga es un transductor que es utilizado para convertir una fuerza en una señal eléctrica. Mediante un dispositivo mecánico, la fuerza que se desea medir deforma el sensor y convierte el desplazamiento o deformación en señales eléctricas. La señal eléctrica de salida es del orden de unos pocos milivolts y debe ser amplificada mediante un amplificador antes de que pueda ser utilizada con cualquier micro controlador que disponga de una entrada ADC. 


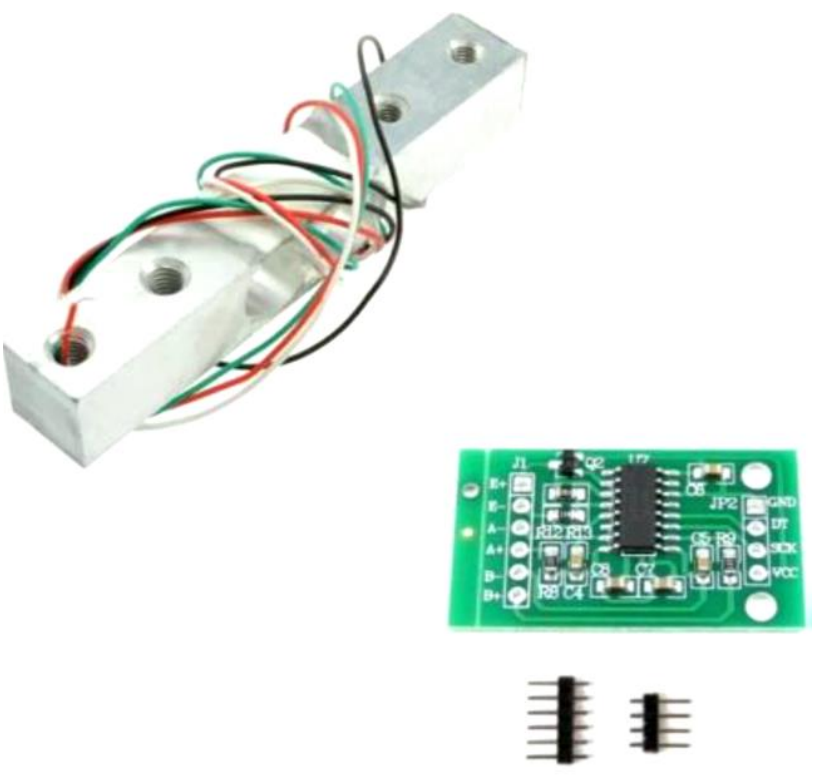

Figura 2 Celda De Carga 5kg Hx711

El DS3231 es un reloj en tiempo real de alta precisión que cuenta con un oscilador a cristal con compensación de temperatura (TCXO). La integración del oscilador a cristal en el propio circuito integrado, en conjunto con la compensación de temperatura, asegura la precisión a largo plazo.

El contador en tiempo real (RTC) mantiene el registro de segundos, minutos, horas, día de la semana, fecha, mes y año, de manera interna, por lo que si requiera colocarse una indicación visual de la hora ya la fecha se tomaría esta informacion del modulo para mostrarlo en una pantalla LCD con dimensiones acordes a la inversion realizada por el consumidor, asi mismo por medio de la programación se ajusta de manera automática los dias para aquellos meses que tengan menos de 31 días, incluyendo las correcciones para año bisiesto.

El modulo RTC DS3231 es capaz de generar señales de reloj cuadradas de frecuencia configurable y además cuenta con 2 alarmas programables que pueden generar interrupciones en el microcontrolador principal en tiempos específicos.

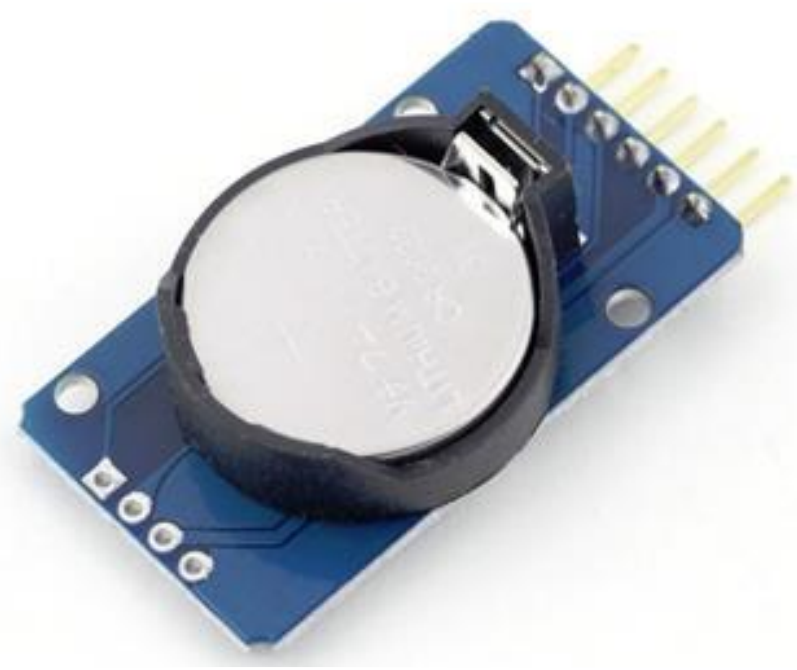

Figura 3 Modulo Reloj De Tiempo Real RTC Ds3231

El módulo Bluetooth HC-06 logra una conexión bidireccional entre dos terminales, por lo que permite controlar el prototipo por medio de un smartphone, o una computadora de forma inalámbrica, previa programacion de una aplicación móvil o software, con la posibilidad de conectarse y desconectarse a todo periferico que cuente con dicha interface (aplicación o software) instalada.

La transmisión se realiza totalmente en forma transparente al programador, por lo que se conecta en forma directa a los pines seriales de nuestro microcontrolador preferido (respetando los niveles de voltaje, ya que el módulo se alimenta con $3.3 \mathrm{~V}$ ). Todos los parámetros del módulo se pueden configurar mediante comandos AT. La placa también incluye un regulador de $3.3 \mathrm{~V}$, que permite alimentar el módulo con un voltaje entre $3.6 \mathrm{~V}-6 \mathrm{~V}$.

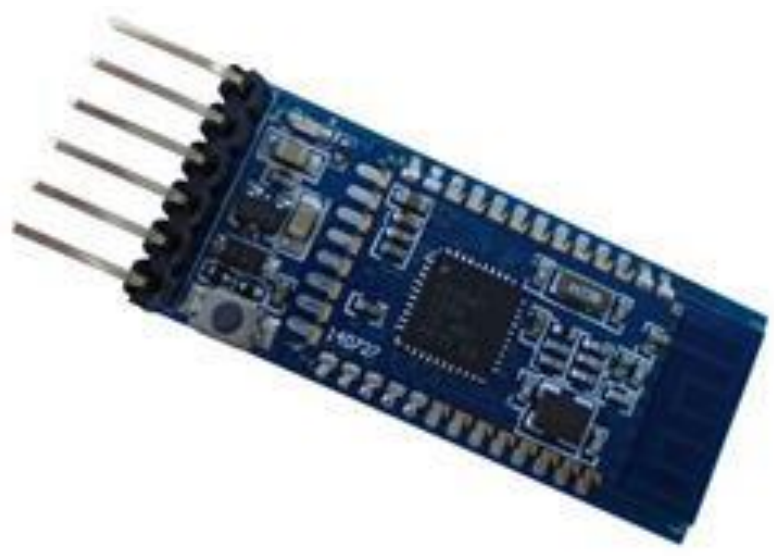

Figura 4 Modulo Bluetooth Hc-06: 
La tarjeta de control Arduino es una placa electrónica es una PCB ("Printed Circuit Board", "Placa de Circuito Impreso" en español). Las PCBs superficies planas fabricadas en un material no conductor, la cual costa de distintas capas de material conductor. Una PCB es la forma más compacta y estable de construir un circuito electrónico.

Así que la placa Arduino no es más que una PCB que implementa un determinado diseño de circuitería interna, de esta forma el usuario final no se debe preocupar por las conexiones eléctricas que necesita el microcontrolador para funcionar, y puede empezar directamente a desarrollar las diferentes aplicaciones electrónicas que necesite.

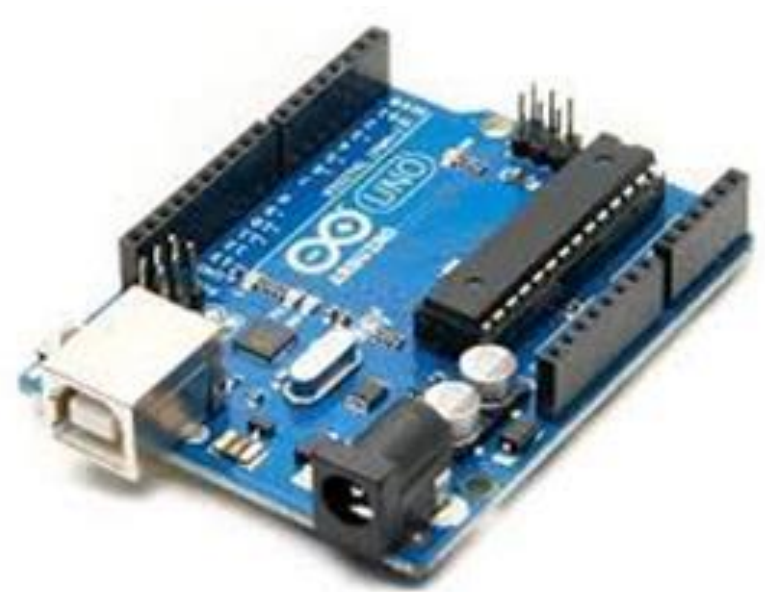

Figura 5 Tarjeta Microcontrador Arduino

El Arduino IDE es un entorno de desarrollo y en él se realiza la programación de cada una de las placas de Arduino. Tiene como base el entorno de Processing al igual que un lenguaje de programación fundamentado en Wiring.

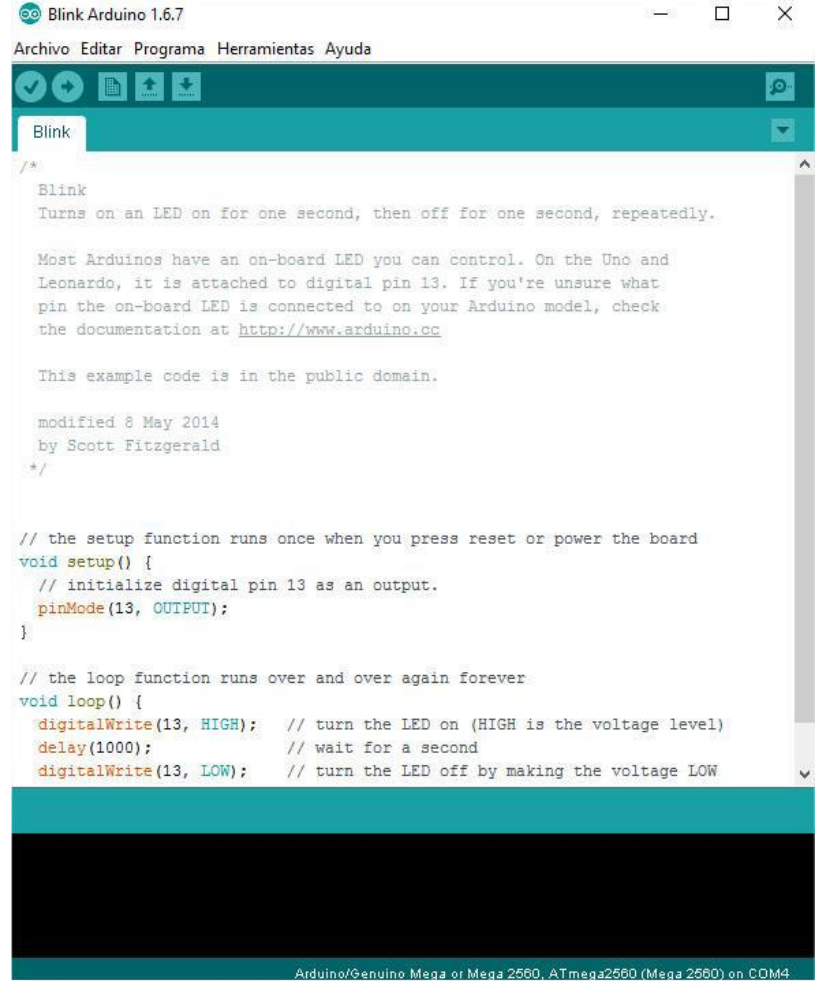

Figura 6 Plataforma Arduino IDE

\section{Estructura y desarrollo del proyecto}

El prototipo en un inicio está realizado con madera, siendo la parte externa una caja de madera con cortes para la entrada y salida del alimento, así como un arreglo para poder mover el circuito de ser necesario. Dentro de la caja esta un contenedor de comida sobre una delgada tabla de madera, en la cual está instalado un servomotor el cual permite o deniega el paso de comida. Debajo de la tabla con el servomotor hay un tubo PVC para la movilidad de la comida y así pueda llegar a su destino en el plato de comida de la mascota. 


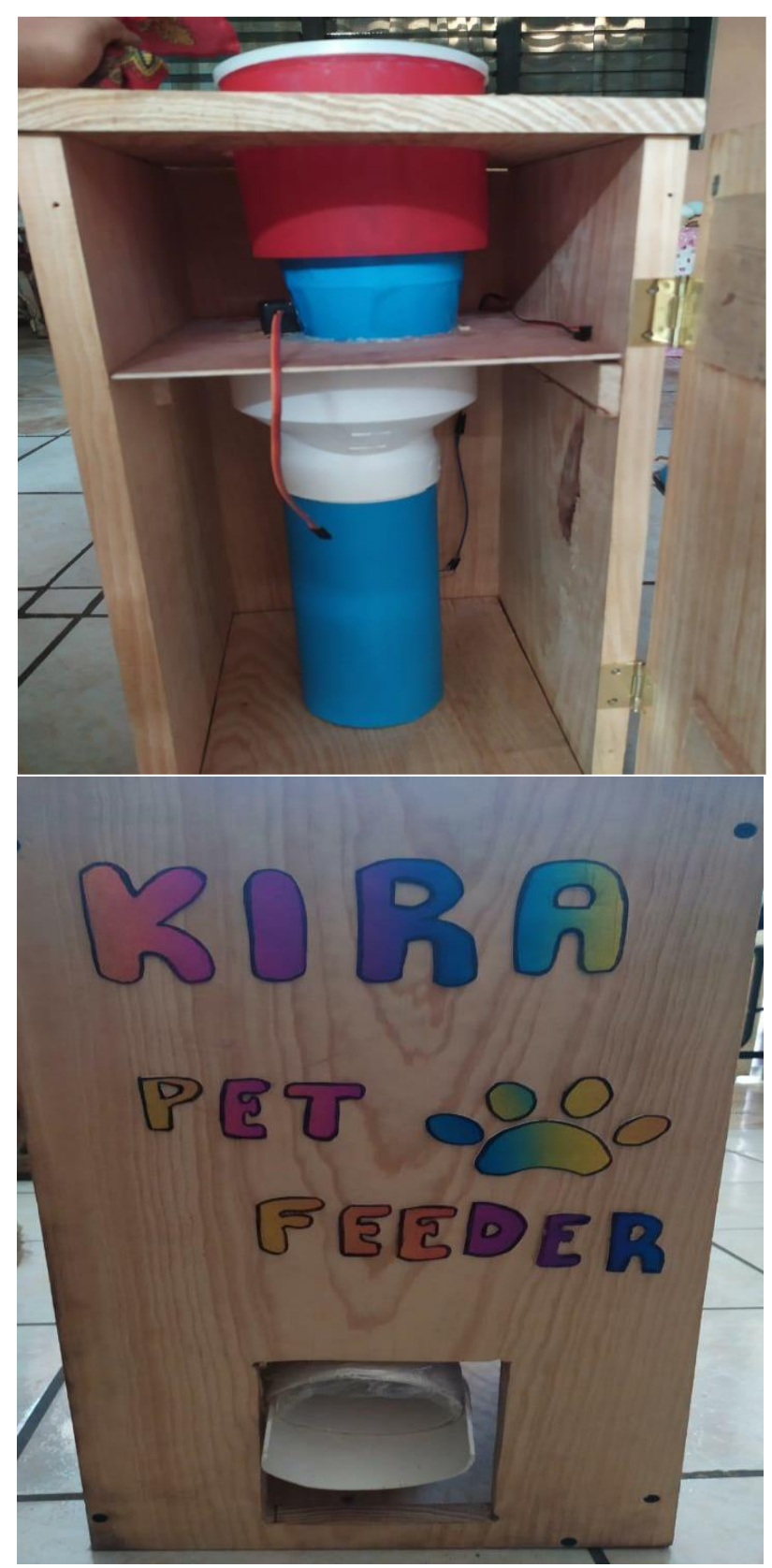

Figura 7 Estructura Física del Prototipo

Mientras que los diagramas eléctricos de las conexiones internas se muestran en las siguientes imágenes, realizando los diagramas de manera individual para su correcta apreciación entre cada uno de los dispositivos que controlan el proceso del alimentador automático.

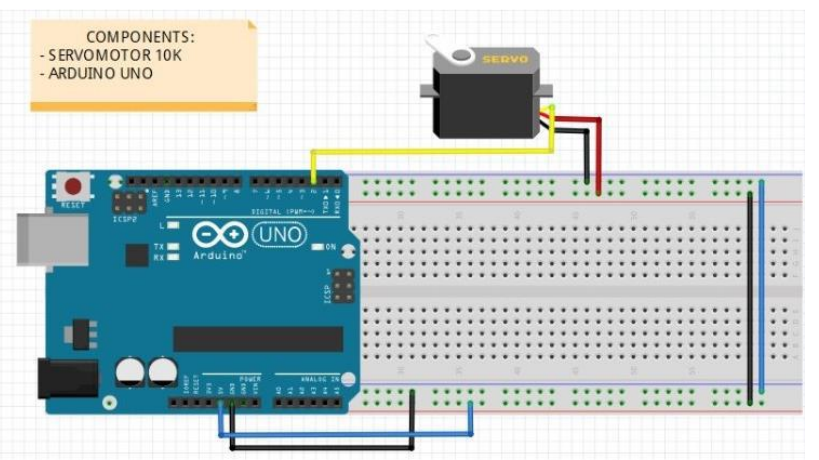

Figura 8 Diagrama del circuito del servomotor

ISSN-2531-2952

ECORFAN $^{\circledR}$ Todos los derechos reservados

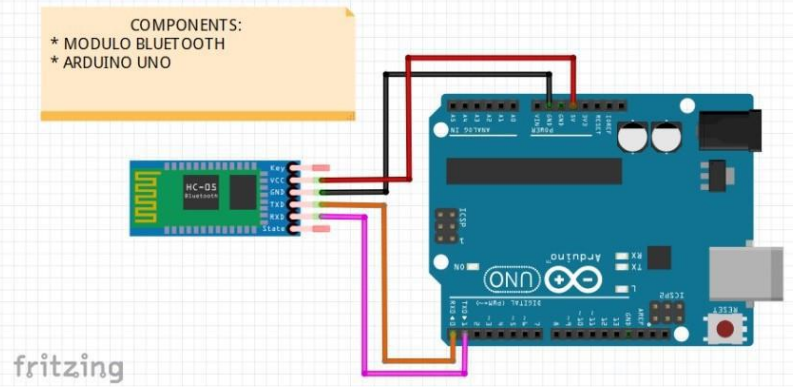

Figura 9 Diagrama del Circuito para el Módulo Bluetooth

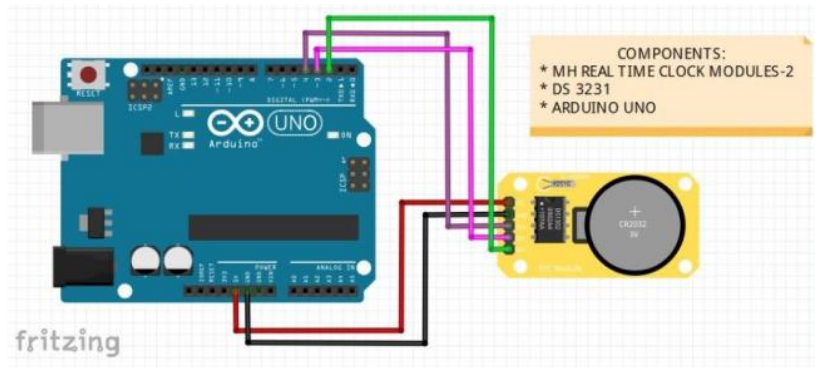

Figura 10 Diagrama del Circuito para Módulo de Reloj en Tiempo Real

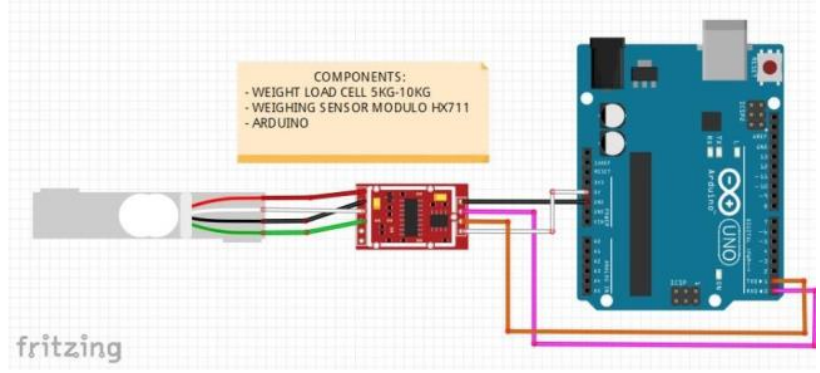

Figura 11 Diagrama del Circuito para Celda de Carga $5 \mathrm{~kg}$

\section{Discusiones y trabajos a futuro}

La maqueta está realizada con madera, con un costo aproximado de $\$ 700.00$, siendo la parte externa una caja de madera con cortes para la entrada y salida del alimento, así como un arreglo para poder mover el circuito de ser necesario. Dentro de la caja esta un contenedor de comida sobre una delgada tabla de madera, en la cual está instalado un servomotor el cual permite o deniega el paso de comida. Debajo de la tabla con el servomotor hay un tubo PVC para la movilidad de la comida y así pueda llegar a su destino en el plato de comida de la mascota.

Un proyecto sumamente útil para nosotros, que en ocasiones salimos de viaje y tenemos que dejar a las mascotas solas y con montones de comida, en donde la mascota no se sabrá medir y comerá más o menos de lo que debería de comer, u ocasiones en las que simplemente no se tiene tiempo de alimentarlas como debería hacerse.

ZAPIEN-RODRIGUEZ, Jose Manuel, CRUZ-BRICEÑO, Guadalupe Janeth, ROSILES-REYES, Carlos Andres y TORRES-CARRILLO, Eva Alejandra. Sistema remoto de alimentacion semiautomatizado para mascotas. Revista de Cómputo Aplicado. 2020 
También, nos dimos cuenta de algunos errores a la hora de planear como sería realizado el proyecto, como el hecho de tener que utilizar más de una celda de carga para poder tener una báscula calibrada y con un funcionamiento adecuado. Algunas de las mejoras que se le planean hacer al proyecto son:

- Adaptarle un módulo WIFI para que el equipo tenga un mayor alcance y sea más efectivo en cuestión de funcionamiento.

- Hacer el prototipo en un tamaño mayor para que la capacidad de almacenamiento de alimento sea mayor.

- Hacer la base con un material más duradero y más liviano como bien puede ser de aluminio por que en ocasiones las mascotas no están entrenadas y pueden orinar o incluso morder el equipo.

- Utilizar dos o cuatro sensores de peso, ya que uno solo no es suficiente para medir el peso de una manera adecuada por problemas de distribución de peso en el recipiente.

\section{Referencias}

Moltoni, A., Moltoni, L., \& Clemares, N. (2020). La Electrónica y los Sistemas Agroindustriales-Nuevo paradigma tecnoproductivo. Electronic Journal of SADIO (EJS), 19(1), 96-117.

Oficial, D. RESOLUCIÓN 957 DE 2018 (mayo 31) Diario Oficial No. 50.611 de 1 de junio de 2018 MINISTERIO DE AMBIENTE Y DESARROLLO SOSTENIBLE.

Chamorro Cadena, D. V. (2020). Análisis multitemporal de los patrones espaciales del paisaje en el cantón Pimampiro, provincia de Imbabura (Bachelor's thesis).

Bazan Santamaria, L. D. (2020). Estrategia de Gestión Académica basada en la Teoría del camino a la Meta de Robert House, para mejorar la Formación Profesional en los estudiantes del IV Ciclo de la Especialidad Matemática y Computación. Facultad de Ciencias Histórico Sociales y Educación-UNPRG-2014.
Huallpa Tarqui, J. (2015). Dosificador automatico diario de alimentos para animales menores.

Castillo Lozada, A. D. L. Á. (2017). Implementación de un sistema automatizado para la selección y dosificación de alimentos para perros en el hogar (Bachelor's thesis, Escuela Superior Politécnica de Chimborazo).

\section{ZAPIEN-RODRIGUEZ, J., RAMIREZ-} CHAVEZ, M., BURGARA-MONTERO, O., \& ESCOTO-SOTELO, E. Desarrollo de un sistema SCADA para monitoreo remoto de grúas RTG en la empresa LCTPC del Puerto de Lázaro Cárdenas, Michoacán, México. Volumen 4, Número 12-Julio-Septiembre-2017, 36.

Sánchez, E. S., Hernández, J. M., \& Calle, M. M. G. Manual para la tenencia responsable de mascotas. Fondo Editorial Biogénesis, 55.

Díaz Eizmendiz, M. (2016). Diseño de sistema domótico de una vivienda mediante un controlador lógico programable (PLC) del tipo TM-251 (Bachelor's thesis, Universitat Politècnica de Catalunya).

Thomas, A. M., Joseph, J., \& Mathew, R. (2016). Mobile Application for Automobile Management System. Journal for Research| Volume, 2(02).

Dandge, J. A., Shirwadkar, R., Gite, P., Odhekar, N., \& Kakad, C. (2016). Electric Switch On/Off System Using Android App Via Wi-Fi. International Research Journal of Engineering and Technology (IRJET), 3(03), 1278-1282.

Ganar, S., Dhote, A., Shivhare, A., Gaikwad, K., \& Mahure, C. (2017). Protection of home using Wireless Bluetooth Technology. International Journal of Engineering Science, 5770.

Tamboli, M., \& Shaikh, F. (2017). Remote access robot via internet.

Pichen, V., \& Polnster, F. (2018). Desarrollo de un prototipo eléctrico para el encendido y apagado de luces con arduino controlado desde una aplicación Android via Bluetooh para la escuela de tecnologías de la información del Senati zonal Ancash-Huaraz; 2018. 